Annals of Pure and Applied Mathematics

Vol. 17, No. 2, 2018, 249-257

ISSN: 2279-087X (P), 2279-0888(online)

Published on 30 June 2018

Annals of

www.researchmathsci.org

DOI: http://dx.doi.org/10.22457/apam.v17n2a11

Pure and Applied

Mathematics

\title{
Degree Based Topological Indices for Buckyball
}

\author{
Thayamathy Pio Jude ${ }^{1}$, Elango Panchadcharam ${ }^{2}$ and Masilamani Koneswaran ${ }^{3}$ \\ ${ }^{1,2}$ Department of Mathematics, Faculty of Science, Eastern University \\ Sri Lanka. E-mail: thayamathypio@gmail.com \\ ${ }^{3}$ Department of Chemistry, Faculty of Science, Eastern University \\ Sri Lanka. E-mail: koneshmas@gmail.com \\ Corresponding author. E-mail: elangop@esn.ac.lk
}

Received 8 May 2018; accepted 29 June 2018

Abstract. Buckyball, a $C_{60}$ molecule, is highly symmetric closed cage structure with a 60 number of carbon atoms and the diameter of 0.78 nanometers. This is formed by twelve pentagons and twenty hexagons that are linked by single and double carbon-carbon bonds. In this paper, we derive the $M$-polynomial for the Buckyball and calculate some of the important degree-based topological indices by using the $M$-polynomial. We also find the forgotten polynomial and the forgotten index ( $F$-index) for the Buckyball.

Keywords: Degree-based topological index, M-polynomial, Buckyball, F-index

\section{AMS Mathematics Subject Classification (2010): 05 C62}

\section{Introduction}

Carbon is the sixth element on the periodic table and is the building block of the universe. Only two allotropes of carbon, namely diamond and graphite were known by the scientific community until Buckyball was accidentally discovered by Curl, Kroto and Smalley in 1985 [11] during carbon nucleation studies under the red giant stars conditions. These three scientists received the Nobel Prize for Chemistry in 1996 for the discovery of this new and fascinating molecular allotropic form of carbon and the elucidation of its unique structure. Buckyball is highly symmetric closed cage structure with a 60 number of carbon atoms and the diameter of its structure is 0.78 nanometers. This $\mathrm{C}_{60}$ molecule is formed by twelve pentagons and twenty hexagons that are linked by single and double carbon-carbon bonds and its structure just like the soccer ball it resembles [2]. Unique shape and electron bonding of buckyball give them outstanding chemical, physical and physicochemical properties and therefore, this molecule has received a lot of attention by the researches during the last two decades [20]. Buckyball can be prepared by many methods. This structure is currently prepared by striking an electrical arc between two graphite electrodes in a helium atmosphere [18]. This allotropic form of carbon has many applications in the fields of chemistry, physics, electronics and medicine [16]. 
Thayamathy Pio Jude, Elango Panchadcharam and Masilamani Koneswaran

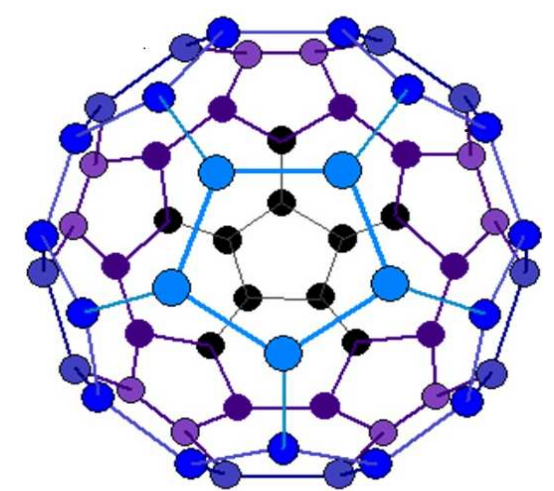

Figure 1: Bucky ball

In this paper we present some important degree based topological indices in the following section and the next section we discuss the degree based topological indices for buckyball.

\section{Some degree based topological indices}

A molecular graph in the molecular graph theory or molecular topology is a simple graph which has neither loops nor multiple edges. In molecular graphs, the atoms and the chemical bonds between them are represented by vertices and edges respectively in the graph theory. A graph $G=G(V, E)$ is a pair $V=V(G)$ of nonempty set of vertices and $E=E(G)$ of the set of connected edges if there exists a connection between any pair of vertices in $G$. For a connected graph $G$ and vertices $u, v$ in the set of vertices $V(G)$, the degree of $v$ is the number of vertices which are connected to that vertex by the edges and is denoted by $d_{v}(G)$ or $d_{v}$. It is noted that the degree of any vertex in a chemical graph is at most four. The concept of degree is closely related to the concept of valence bond in chemistry. The distance between two vertices $u$ and $v$ is the length of shortest path between these vertices and is denoted by $d(u, v)$ or $d_{G}(u, v)$.

A topological index is a numerical quantity which is derived mathematically in a direct and unambiguous manner from the structural graph of a molecule. Many properties of a chemical compound are closely related to some topological indices of its molecular graph. The Wiener index is the first and the most studied distance-based topological index in chemical graph theory. This index was introduced by the chemist H. Wiener [19] in 1947 to demonstrate correlations between physicochemical properties of organic compounds and the topological structure of their molecular graphs. He defined this index as

$$
W(G)=\sum_{\{u, v\} \subseteq V(G)} d_{G}(u, v),
$$

the sum of distances between all the carbon atoms in the molecules, in terms of carboncarbon bonds and himself used the name path number.

Among the different type of topological indices, the degree-based topological indices are the most studied type of topological indices which play a prominent role in chemical graph theory. One of the oldest degree-based topological indices is the well- 


\section{Degree Based Topological Indices for Buckyball}

known Zagreb index, introduced by Gutman and Trinajstic [9] in 1972 during the analysis of the structure-dependency of total $\pi$-electron energy. The name Zagreb is the place in which both of them worked in an institute as members of the theoretical chemistry group. The first Zagreb index $M_{1}(G)$ is defined as

$$
M_{1}(G)=\sum_{v \in V(G)}\left(d_{v}\right)^{2}=\sum_{u v \in E(G)}\left(d_{u}+d_{v}\right) .
$$

The second Zagreb index $M_{2}(G)$ is defined as

$$
M_{2}(G)=\sum_{u v \in E(G)} d_{u} d_{v} .
$$

The second modified Zagreb index [10] is defined as

$$
{ }^{m} M_{2}(G)=\sum_{u v \in E(G)} \frac{1}{d_{u} d_{v}}
$$

The multiplicative versions of this Zagreb index for certain nanotubes were calculated by Kulli [12]. The Randić index which is also one of the oldest topological indices was introduced by Milan Randić [17] in 1974 and was defined as Randić

$$
R(G)=\sum_{u v \in E(G)} \frac{1}{\sqrt{d_{u} d_{v}}}
$$

Randić himself named this index as branching index and soon it was renamed as connectivity index. Nowadays, it refers as the Randić index. The Randic index is the most studied, most often applied and most popular degree-based topological index which is used in the field of drug design. Many papers and books are written on this Randic index. Later this index was globalized and recognized as the generalized Randić index defined as

$$
R_{\alpha}(G)=\sum_{u v \in E(G)}\left(d_{u} d_{v}\right)^{\alpha} .
$$

where $\alpha$ is an arbitrary real number [15]. When, $\alpha=-1 / 2$ the above formula becomes the (original) Randić index.

The following formula is also called the generalized Randić index [3] and defined as

$$
R R_{\alpha}(G)=\sum_{u v \in E(G)} \frac{1}{\left(d_{u} d_{v}\right)^{\alpha}}
$$

The atom-based connectivity index abbreviated by $A B C$-index was introduced by Ernesto Estrada [4]. This is an amended version of the Randić index and is defined as

$$
A B C(G)=\sum_{u v \in E(G)} \sqrt{\frac{d_{u}+d_{v}-2}{d_{u} d_{v}}} .
$$

The $A B C$-index has excellent correlation with the thermodynamic properties of alkanes, especially with their heats of formation. Some multiplicative $A B C$-indices for certain nanostructures were introduced by Kulli $[13,14]$. 
Thayamathy Pio Jude, Elango Panchadcharam and Masilamani Koneswaran

The success of the $A B C$-index led to the invention of the augmented Zagreb index which was introduced by Furtula et al [8] and defined as

$$
A(G)=\sum_{u v \in E(G)}\left\{\frac{d_{u} d_{v}}{d_{u}+d_{v}-2}\right\}^{3} .
$$

This index is useful for computing heat formation of alkanes. It is noted that the $A B C$-index can be derived from this augmented Zagreb index by replacing its power 3 by the power -0.5 .

Fajtlowicz [5] first introduced a vertex-degree-based quantity and later it was reintroduced as the harmonic index and defined as

$$
H(G)=\sum_{u v \in E(G)} \frac{2}{d_{u}+d_{v}}
$$

as another variant of the Randic index. Favaron et al [6] showed the relation between the harmonic index and the eigen values of graphs.

The inverse sum index [1] is a significant predictor of the total surface area of octane isomers which is defined as

$$
I(G)=\sum_{u v \in E(G)} \frac{d_{u} d_{v}}{d_{u}+d_{v}}
$$

The symmetric division index is used to characterize the chemical and physical properties of molecules and defined as

$$
S D D(G)=\sum_{u v \in E(G)}\left\{\frac{\min \left(d_{u}, d_{v)}\right.}{\max \left(d_{u}, d_{v}\right)}+\frac{\max \left(d_{u}, d_{v)}\right.}{\min \left(d_{u}, d_{v)}\right.}\right\} .
$$

The forgotten topological index or $F$-index has more applications in the analysis of drug molecular structures, which was introduced by Furtula and Gutman [7]. The Findex is used to express the biological and chemical characteristics of new drugs. The $F$ polynomial $F(G, x)$ and the $F$-index $F(G)$ of a graph $G$ are defined as

and

$$
F(G, x)=\sum_{u v \in E(G)} x^{\left(d_{u}^{2}+d_{v}^{2}\right)}
$$

$$
F(G)=\sum_{v \in V(G)}\left(d_{v}\right)^{3}=\sum_{u v \in E(G)}\left(d_{u}^{2}+d_{v}^{2}\right) .
$$

Many graph polynomials play a prominent role in mathematical chemistry to study the structural properties of the molecules. Among them, the Hosoya polynomial (also known as the Wiener polynomial) plays a vital role to determine the distance-based topological indices. In particular, the first derivative of the Hosoya polynomial can be used to compute the Wiener index. Other polynomials are the matching polynomial, the Zhang-Zhang polynomial, Schultz polynomial and the Tutte polynomial are a few of them. Another important polynomial is the $M$-polynomial which was introduced in 2015 by Deutsch and Klavzar [3]. The $M$-polynomial plays another important role to determine many degree-based topological indices. That is, it plays the role to determine the degree- 
Degree Based Topological Indices for Buckyball

based topological indices as the role of the Hosoya polynomial for the determination of the distance-based topological indices.

Theorem 2.1. ([3]) Let $G=G(V, E)$ be a graph and let $m_{i j}(G), i, j \geq 1$, be the number of edges $e=u v$ of $G$ such that $\left\{d_{u}, d_{v}\right\}=\{i, j\}, u, v \in V(G)$. Then the $M$ polynomial of $G$ is

$$
M(G ; x, y)=\sum_{i \leq j} m_{i j}(G) x^{i} y^{j} .
$$

Some of the degree-based topological indices were derived from $M$-polynomial and given in the following table:

Table 1: Some standard degree-based topological indices and the derived formulas to compute them from the $M$-polynomial ([3]).

\begin{tabular}{|l|c|l|}
\hline \multicolumn{1}{|c|}{ Topological index } & $\boldsymbol{f}(\boldsymbol{x}, \boldsymbol{y})$ & \multicolumn{1}{c|}{ Derivation from $\boldsymbol{M}(\boldsymbol{G} ; \boldsymbol{x}, \boldsymbol{y})$} \\
\hline $\begin{array}{c}\text { General Randić } \\
(\alpha \in \mathbb{N})\end{array}$ & $(x y)^{\alpha}$ & $\left(D_{x}^{\alpha} D_{y}^{\alpha}\right)(M(G ; x, y))_{x=y=1}$ \\
\hline $\begin{array}{l}\text { General Randić } \\
(\alpha \in \mathbb{N})\end{array}$ & $\frac{1}{(x y)^{\alpha}}$ & $\left(S_{x}^{\alpha} S_{y}^{\alpha}\right)(M(G ; x, y))_{x=y=1}$ \\
\hline First Zagreb & $x+y$ & $\left(D_{x}+D_{y}\right)(M(G ; x, y))_{x=y=1}$ \\
\hline Second Zagreb & $\frac{1}{x y}$ & $\left(D_{x} D_{y}\right)(M(G ; x, y))_{x=y=1}$ \\
\hline $\begin{array}{l}\text { Second modified } \\
\text { Zagreb }\end{array}$ & $\left(\frac{x y}{x+y-2}\right)^{3}$ & $S_{x}^{3} Q_{-2} J D_{x}^{3} D_{y}^{3}(M(G ; x, y))_{x=y=1}$ \\
\hline $\begin{array}{l}\text { Augmented Zagreb } \\
\text { index }\end{array}$ & & \\
\hline
\end{tabular}

Table 2: Some standard degree-based topological indices and the derived formulas to compute them from the $M$-polynomial ([3]).

\begin{tabular}{|l|c|l|}
\hline Topological index & $\boldsymbol{f}(\boldsymbol{x}, \boldsymbol{y})$ & Derivation from $\boldsymbol{M}(\boldsymbol{G} ; \boldsymbol{x}, \boldsymbol{y})$ \\
\hline Harmonic index & $\frac{2}{x+y}$ & $2 S_{x} J(M(G ; x, y))_{x=1}$ \\
\hline Inverse sum index & $\frac{x y}{x+y}$ & $S_{x} J D_{x} D_{y}(M(G ; x, y))_{x=1}$ \\
\hline $\begin{array}{l}\text { Symmetric division } \\
\text { index }\end{array}$ & $\frac{x^{2}+y^{2}}{x y}$ & $\left(D_{x} S_{y}+S_{x} D_{y}\right)(M(G ; x, y))_{x=y=1}$ \\
\hline
\end{tabular}

where 
Thayamathy Pio Jude, Elango Panchadcharam and Masilamani Koneswaran

$D_{x}=x \frac{\partial f(x, y)}{\partial x}, D_{y}=y \frac{\partial f(x, y)}{\partial y} S_{x}=\int_{0}^{x} \frac{f(t, y)}{t} d t, \quad S_{y}=\int_{0}^{y} \frac{f(x, t)}{t} d t$,

$J(f(x, y))=f(x, x)$ and $Q_{\alpha}(f(x, y))=x^{\alpha} f(x, y)$.

\section{Result and discussion}

We compute the degree-based topological indices of buckyball using the $M$-polynomial for this buckyball. Later, we calculate other degree-based topological indices directly from the formulas. The buckyball $\mathrm{C}_{60}$ with sixty carbon atoms is shown in the Figure 1.

Theorem 3.1. Let $G=C_{60}$ be the buckyball. Then the $M$-polynomial for the buckyball is given by

$$
M\left(C_{60}, x, y\right)=90 x^{3} y^{3} .
$$

Proof: The buckyball $G=C_{60}$ is given in the figure 1 . This has 60 carbon atoms and 90 carbon-carbon bonds. Therefore, the numbers of vertices $v$ is 60 and edges $u v$ are 90 . All these 60 vertices are with the degree 3. That is, $V\left(C_{60}\right)=\left\{v \in C_{60}: d v=3\right\}$.

Therefore, the $M$-polynomial of $G=C_{60}$ is

$$
M\left(C_{60}, x, y\right)=\sum_{i \leq j} m_{i j} x^{i} y^{j}=m_{33} x^{3} y^{3}=90 x^{3} y^{3}
$$

Proposition 3.1. Let $G=C_{60}$ be the buckyball, then the degree-based topological indices for $C_{60}$ are

1. $M_{1}\left(C_{60}\right)=540$.

2. $M_{2}\left(C_{60}\right)=810$.

3. ${ }^{m} M_{2}\left(C_{60}\right)=10$.

4. $R_{\alpha}\left(C_{60}\right)=90 \times 9^{\alpha}$.

5. $R R_{\alpha}\left(C_{60}\right)=\frac{90}{9^{\alpha}}$.

6. $\operatorname{SDD}\left(c_{60}\right)=180$.

Proof: Let $f(x, y)$ be the $M$-polynomial of $C_{60}$. Then, $f(x, y)=90 x^{3} y^{3}$ and we get the following

$$
\begin{gathered}
D_{x}(f(x, y))=270 x^{3} y^{3}, D_{y}(f(x, y))=270 x^{3} y^{3}, D_{x} D_{y}(f(x, y))=810 x^{3} y^{3}, \\
S_{x}(f(x, y))=30 x^{3} y^{3}, S_{y}(f(x, y))=30 x^{3} y^{3}, S_{x} S_{y}(f(x, y))=10 x^{3} y^{3}, \\
D_{y}^{\alpha}(f(x, y))=90 \times 3^{\alpha} x^{3} y^{3}, D_{x}^{\alpha} D_{y}^{\alpha}(f(x, y))=90 \times 9^{\alpha} x^{3} y^{3}, S_{y}^{\alpha}(f(x, y))= \\
\frac{90}{3^{\alpha}} x^{3} y^{3}, S_{x}^{\alpha} S_{y}^{\alpha}(f(x, y))=\frac{90}{9^{\alpha}} x^{3} y^{3}, D_{x} S_{y}(f(x, y))=90 x^{3} y^{3}, \\
S_{x} D_{y}(f(x, y))=90 x^{3} y^{3} .
\end{gathered}
$$

By using the derived formulas of $M$-polynomial given in the table 1 , we find

1. $M_{1}\left(C_{60}\right)=\left.\left(D_{x}+D_{y}\right)(f(x, y))\right|_{x=y=1}=540$.

2. $M_{2}\left(C_{60}\right)=\left.D_{x} D_{y}(f(x, y))\right|_{x=y=1}=810$.

3. ${ }^{m} M_{2}\left(C_{60}\right)=\left.S_{x} S_{y}(f(x, y))\right|_{x=y=1}=10$. 
Degree Based Topological Indices for Buckyball

4. $R_{\alpha}\left(C_{60}\right)=\left.D_{x}^{\alpha} D_{y}^{\alpha}(f(x, y))\right|_{x=y=1}=90 \times 9^{\alpha}$.

5. $R R_{\alpha}\left(C_{60}\right)=\left.S_{x}^{\alpha} S_{y}^{\alpha}(f(x, y))\right|_{x=y=1}=\frac{90}{9^{\alpha}}$.

6. $S D D\left(c_{60}\right)=\left.\left(D_{x} S_{y}+S_{x} D_{y}\right)(f(x, y))\right|_{x=y=1}=180$.

Proposition 3.2. Let $G=C_{60}$ be the buckyball, then

1. $H\left(C_{60}\right)=30$

2. $I\left(C_{60}\right)=135$

3. $A\left(C_{60}\right)=\frac{32805}{32}$

Proof: We have $M$-polynomial of $C_{60}$ is $f(x, y)=9 x^{3} y^{3}$. Then we get the following $J(f(x, y))=90 x^{6}, S_{x} J(f(x, y))=15 x^{6}, J D_{x} D_{y}(f(x, y))=810 x^{6}$, $S_{x} J D_{x} D_{y}(f(x, y))=135 x^{6}, D_{y}^{3}(f(x, y))=810 x^{3} y^{3}, D_{x}^{3} D_{y}^{3}(f(x, y))=90 \times$ $3^{6} x^{3} y^{3}, J D_{x}^{3} D_{y}^{3}(f(x, y))=90 \times 3^{6} x^{6}$, $Q_{-2} J D_{x}^{3} D_{y}^{3}(f(x, y))=90 \times 3^{6} x^{4}, S_{x}^{3} Q_{-2} J D_{x}^{3} D_{y}^{3}(f(x, y))=\frac{90 \times 3^{6}}{4^{3}} x^{4}$.

By using the derived formulas of $M$-polynomial given in the table 2 , we find

1. $H\left(C_{60}\right)=\left.2 S_{x} J(f(x, y))\right|_{x=y=1}=30$.

2. $I\left(C_{60}\right)=\left.S_{x} J D_{x} D_{y}(f(x, y))\right|_{x=y=1}=135$.

3. $A\left(C_{60}\right)=\left.S_{x}^{3} Q_{-2} J D_{x}^{3} D_{y}^{3}(f(x, y))\right|_{x=y=1}=\frac{32805}{32}$.

Proposition 3.3. The derivative formula for the Atom-Bond Connectivity index for $C_{60}$, $A B C\left(C_{60}\right)$ from the $M$-polynomial is

$$
\left.S_{x}^{-1 / 2} Q_{-2} J D_{x}^{-1 / 2} D_{y}^{-1 / 2} M(G ; x, y)\right|_{x=y=1} .
$$

Proof: Using the definition of Atom-Bond Connectivity $(A B C)$ index, we get the $A B C$ index for the buckyball $C_{60}$ is

$$
A B C\left(C_{60}\right)=\sum_{u v \in E(G)} \sqrt{\frac{d_{u}+d_{v}-2}{d_{u} d_{v}}}=90 \times \sqrt{\frac{3+3-2}{3 \times 3}}=60 .
$$

We now use our proposition to calculate the $A B C$ index. The derived formulas of $M$ polynomial are

$$
\begin{gathered}
D_{y}^{-1 / 2}(f(x, y))=30 \sqrt{3} x^{3} y^{3}, D_{x}^{-1 / 2} D_{y}^{-1 / 2}(f(x, y))=30 x^{3} y^{3}, \\
J D_{x}^{-1 / 2} D_{y}^{-1 / 2}(f(x, y))=30 x^{6}, Q_{-2} J D_{x}^{-1 / 2} D_{y}^{-1 / 2}(f(x, y))=30 x^{4} \\
S_{x}^{-1 / 2} Q_{-2} J D_{x}^{-1 / 2} D_{y}^{-1 / 2}(f(x, y))=60 x^{4} .
\end{gathered}
$$

Therefore, the $A B C$ index for the buckyball $C_{60}$ is

$$
\left.A B C\left(C_{60}\right) S_{x}^{-1 / 2} Q_{-2} J D_{x}^{-1 / 2} D_{y}^{-1 / 2}(f(x, y))\right|_{x=y=1}=60 .
$$


Thayamathy Pio Jude, Elango Panchadcharam and Masilamani Koneswaran

This shows that the derived formula of M-polynomial proposed in the Proposition 3.3 for the $\mathrm{ABC}$ index is correct.

Theorem 3.2. The $F$-polynomial and the $F$-index for the buckyball $C_{60}$ are

$$
F\left(C_{60}, x\right)=90 x^{18} \text { and } F\left(C_{60}\right)=1620 \text {. }
$$

Proof: Let $G=C_{60}$. Then we have, $d_{v}=3$ for each $v \in C_{60}$ and also we have a total of $u v$ in $C_{60}$ is 90 . Substituting these values in the above equations, we get

$$
\begin{gathered}
F\left(C_{60}, x\right)=\sum_{u v \in E\left(C_{60}\right)} x^{\left(d_{u}^{2}+d_{v}^{2}\right)}=90 x^{18} . \text { and } \\
F\left(C_{60}\right)=\sum_{u v \in E\left(C_{60}\right)}\left(d_{u}^{2}+d_{v}^{2}\right)=90 \times 18=1620 .
\end{gathered}
$$

\section{Conclusion}

In this paper, we study the structure of buckyball and established some well-known degree based topological indices for buckyball. We also established the F-index and Fpolynomial for the buckyball.

Acknowledgement. Authors are grateful to the reviewers for their valuable suggestions.

\section{REFERENCES}

1. A.T.Balban, Highly discriminating distance based numerical descriptor, Chem. Phys. Lett., 89 (1982) 399-404.

2. J.L.Delgado, S.Filippone, F.Giacalone, M.A.Herranz, B.Illescas, E.M.Perez and N.Martín, Buckyballs, Top Curr Chem., 350 (2014) 1-64.

3. E.Deutsch and S.Klavzar, M-polynomial and degree-based topological indices, Iran. J. Math. Chem., 6 (2015) 93-102.

4. E.Estrada, L.Torres, L.Rodriguez and I.Gutman, An atombond connectivity index: Modelling the enthalpy of formation of alkanes, Indian J. Chem., 37A (1998) 849855.

5. S.Fajtlowicz, On conjectures of GraffitiII, Congr. Numer., 60 (1987) 187-197.

6. O.Favaron, M.Maho and J.F.Sacl, Some eigenvalue properties in graphs conjectures of Graffiti-II, Discrete Math., 111 (1993) 197-220.

7. B.Furtula and I.Gutman, A fogotten topological index, J. Math. Chem., 86 (2015) 1184-1190.

8. B.Furtula, A.Graovac and D.Vuki `ević, Augmented Zagreb index, J. Math. Chem. 48 (2010) 370-380.

9. I.Gutman and N.Trinajstić Graph theory and molecular orbitals total $f$-elecron energy of alternant hydrocarbons, Chem.Phys. Lett., 77 (1972) 535-538.

10. J.Hao, Theorems about Zagreb Indices and Modified Zagreb Indices, MATCH Commun. Math. Comput. Chem., 65 (2011) 659-670.

11. H.W.Kroto, J.R.Heath, S.C.O'Brien, R.F.Curl and R.E.Smalley, C60: Buck minsterfullerene, Nature, 318 (1985) 162-163.

12. V.R.Kulli, Multiplicative connectivity indices of certain nanotubes, Annals of Pure and Applied Mathematics, 12 (2016) 169-176. 
Degree Based Topological Indices for Buckyball

13. V.R.Kulli, Two new multiplicative atom bond connectivity indices, Annals of Pure and Applied Mathematics, 13 (2017) 1-7.

14. V.R.Kulli, Multiplicative atom-bond connectivity and multiplicative geometricarithmetic indices of dendrimer nanostars, Annals of Pure and Applied Mathematics, 16 (2018) 429-436.

15. X.Li and Y.Shi, A Survey on the Randi'c Index, MATCH Commun. Math. Comput. Chem., 59 (2008) 127-156.

16. N.Martín, New challenges in fullerene chemistry, Chem Commun., 20 (2006) 20932104.

17. M.Randić, On Characterization of molecular branching, J. Am. Chem. Soc., 97 (1974) 6609-6615.

18. C.Talbot, Fullerene and nanotube chemistry: An update, School Science Review, 81 (1999) 37-48.

19. H.J.Wiener, Structural determination of Paraffin boiling points, J. Amer. Chem. Soc., 69 (1947) 17-20.

20. B.C.Yadav and R.Kumar, Structure, properties and applications of fullerenes, International Journal of Nanotechnology and Applications, 2 (2008) 15-24. 